\section{Como a posição social influencia a auto- avaliação do estado de saúde? Uma análise comparativa entre 1998 e 2003}

\author{
How does social position influence self-reported \\ health status? A comparative analysis between \\ 1998 and 2003
}

\author{
1 Centro de Desenvolvimento \\ e Planejamento Regional, \\ Universidade Federal de \\ Minas Gerais, Belo Horizonte, \\ Brasil. \\ 2 Instituto de Economia, \\ Universidade Estadual de \\ Campinas, Campinas, Brasil. \\ Correspondência \\ C. G. Rodrigues \\ Centro de Desenvolvimento \\ e Planejamento Regional, \\ Departamento de Demografia, \\ Universidade Federal de \\ Minas Gerais. \\ Av. Antonio Carlos 6627, 3 o \\ andar, Belo Horizonte, \\ MG 31270-901, Brasil. \\ cristina@cedeplar.ufmg.br
}

\begin{abstract}
The aim of this paper is to analyze how the social position of families affects self-reported health status, based on data from the 1998 and 2003 National Sample Household Survey of the Brazilian Institute of Geography and Statistics (PNADIBGE). The method was based on descriptive statistics and logistic regression to capture the conditional relationship between health status, social position, and other control variables, such as age, sex, race/color, income, education, and place of residence. The results show that the same hierarchy of the occupational classes is reproduced in self-reported health status. There was an increase in inequalities in the period, with a reduction in the prevalence of healthy workers in the lower social strata and an increase in the upper strata. Although there were more healthy individuals in non-agricultural classes, the probability of good self-reported health was higher among agricultural workers, after the other control variables were added to the regression models. The results emphasize the need to analyze health inequalities from the perspective of social stratification.
\end{abstract}

SocialClass;Self-Rated Health;Health Inequalities
Cristina Guimarães Rodrigues 1 Alexandre Gori Maia ${ }^{2}$

\section{Introdução}

É notória a relação entre medidas de saúde e variáveis sócio-econômicas, estudada segundo diferentes abordagens teóricas. Do ponto de vista econômico, por exemplo, isso se deve à importância da saúde como um determinante fundamental da capacidade produtiva das pessoas 1,2 . Ter boa saúde significa estar mais disposto a exercer atividades dentro e fora do mercado de trabalho. No campo sociológico, os estudos focam a interação entre condições de saúde e fatores socioculturais e biossociais, como status marital, religião, posição na família, raça, gênero, entre outros 3,4. Esses fatores refletem a posição do indivíduo na sociedade e condicionam sua forma de viver e interagir, com conseqüências para a saúde. De forma análoga, a epidemiologia social procura analisar como os determinantes sócio-econômicos e comportamentais influenciam a distribuição de doenças no âmbito populacional 5,6.

A despeito do arcabouço teórico utilizado, é amplamente difundida a importância dos determinantes sociais sobre as condições de saúde de indivíduos e populações 7,8. Determinantes esses dados por fatores econômicos, educacionais, sociais, culturais, raciais, psicológicos e comportamentais, que influenciam a ocorrência de doenças na população e sua exposição a fatores de risco ${ }^{9}$. Eles se desdobram, ainda, em valorações subjetivas distintas do estado de 
saúde, decorrentes não apenas do conhecimento de aspectos clínicos e funcionais do processo saúde-doença, mas de experiências sociais e estilos de vida 10 .

Sabe-se, por exemplo, que a vulnerabilidade associada às ocupações menos qualificadas e de baixos rendimentos possui conseqüências deletérias para a saúde física e emocional das pessoas 11,12. Ocupações de baixo prestígio social, que requerem poucas habilidades ou que demandam maior controle sobre os trabalhadores usualmente associam-se a uma maior exposição aos riscos para a saúde e maior incidência de doenças 13 . Rendimentos menores limitam os gastos relacionados a uma vida saudável, como alimentação rica em nutrientes e condições adequadas de moradia. Da mesma forma, indivíduos inseridos em contextos sociais menos favorecidos tendem a apresentar hábitos mais nocivos à saúde, como fumo e alcoolismo, e maior incidência de comportamentos violentos, além de apresentarem menor participação cívica e coesão social na comunidade em que vivem 8,14,15,16. Esses comportamentos e privações são fonte de frustrações, estresse e sintomas depressivos, que prejudicam a saúde de indivíduos e seus familiares 17 .

Em geral, as evidências sobre a relação entre saúde e status sócio-econômico focam a associação entre rendimentos e estado de saúde 1,18,19,20. Menos freqüentes são os estudos que se propõem a verificar a relação primária entre a saúde e ocupação 6,12,21,22. Nesta perspectiva, são ainda mais escassos os trabalhos que analisam a relação entre a saúde e a posição social das famílias, uma proxy para o comportamento de classes de uma sociedade 23 .

Embora a renda possa definir a inserção dos indivíduos no mercado de bens e produtos de uma sociedade, não pode ser vista como fator delimitador da posição social dos indivíduos. Como alternativa à estratificação da população segundo faixas de rendimentos, a literatura social propõe tipologias baseadas em conceitos mais abrangentes, os quais seriam aproximações mais condizentes ao comportamento de classes de uma sociedade $24,25,26,27$.

Neste trabalho, a posição social dos integrantes familiares baseou-se no método de estratificação proposto por Quadros 28, uma aproximação da divisão social do trabalho elaborada a partir de informações das pesquisas domiciliares. Esse método parte do pressuposto de que grupos sociais com estilos de vida relativamente homogêneos podem ser obtidos a partir da combinação entre a inserção dos integrantes familiares no mercado de trabalho (classes ocupacionais) e os rendimentos familiares per capita (estratos sociais).
A estrutura social, como indicador da posição social das pessoas, permitiria avaliar de que forma os diferentes grupos sociais estariam expostos a riscos e percepções diferenciadas sobre o estado de saúde. Pode-se, por exemplo, supor que famílias pertencentes aos grupos sociais menos favorecidos estejam mais vulneráveis às tensões físicas ou emocionais, associadas à instabilidade sócio-econômica de suas posições. A posição social de uma família seria ainda um indicador mais eficiente do que a renda em análises realizadas em um determinado ponto do tempo, dada a elevada volatilidade desta no curto prazo 29.

Neste contexto, o presente trabalho pretende responder à seguinte pergunta: existem diferenças na percepção do estado de saúde entre indivíduos de posições sociais distintas na hierarquia de classes brasileira? Para responder essa questão, foram utilizadas informações do suplemento de saúde da Pesquisa Nacional por Amostra de Domicílios (PNAD) do Instituto Brasileiro de Geografia e Estatística (IBGE), dos anos de 1998 e 2003. O método baseou-se em tabulações do estado de saúde auto-reportado segundo características sócio-econômicas diversas e coeficientes de ajustes de regressão logística para captar o efeito isolado da posição social sobre a saúde das pessoas. Os resultados pretendem contribuir para o debate acerca da importância dos determinantes sociais sobre a saúde, a partir de indicadores das condições sócio-econômicas não tradicionais, como a posição social. Entendemos que esse debate amplia o conhecimento das circunstâncias e mecanismos sociais que afetam a saúde física e emocional das pessoas.

\section{Materiais e métodos}

\section{Universo de estudo}

As análises deste trabalho basearam-se em informações dos microdados da PNAD dos anos de 1998 e 2003, que contêm suplementos especiais sobre saúde. Seus suplementos permitem analisar, conjuntamente às informações sócio-econômicas do questionário básico, informações de interesse específico, como as condições de saúde da população.

Em 1998, a PNAD investigou informações sobre 90.913 domicílios e 344.975 pessoas, que correspondiam a um universo de aproximadamente 46 milhões de domicílios e 158 milhões de brasileiros. Em 2003, foram investigados 107.846 domicílios e 384.834 pessoas, correspondendo a um universo de aproximadamente 50 milhões de domicílios e 176 milhões de pessoas, que correspondem ao universo pesquisado neste trabalho. 
Mensuração do estado de saúde

A variável de interesse principal (dependente) adotada refere-se à auto-avaliação do estado de saúde, pertencente ao módulo do suplemento de saúde da PNAD de 1998 e 2003. A pergunta que se faz em ambos os questionários a esse respeito é: “De um modo geral, considera seu próprio estado de saúde como...”; com cinco categorias de resposta: "muito boa", "boa", "regular", "ruim" e "muito ruim". Essa variável foi dicotomizada em duas categorias, dividindo os indivíduos em saudáveis (aqueles que reportaram seu estado de saúde como "muito bom" ou "bom") e não saudáveis (respostas "regular", "ruim" ou "muito ruim").

Embora a dicotomização do estado de saúde auto-avaliado implique perda de informação, é a solução freqüentemente adotada pela literatura para simplificar a apresentação e interpretação dos resultados 30,31. Segundo a tipologia proposta, 79,1\% dos indivíduos da população se enquadravam como saudáveis em 1998 e 78,6\% em 2003.

\section{Estratificação sócio-ocupacional}

Segundo Mills ${ }^{32}$, a divisão social do trabalho passou a cumprir papel essencial na formação da estrutura das sociedades capitalistas modernas, sendo o elemento marcante desse processo o surgimento de uma importante massa de trabalhadores assalariados de classe média. A idéia central é que, embora a renda defina a inserção dos indivíduos no mercado de bens e produtos de uma sociedade, ela não pode ser vista como fator delimitador do estilo de vida dos indivíduos. A posição ocupacional dos integrantes familiares, por sua vez, além de definir, em grande medida, a probabilidade de geração presente e futura de renda da família, estaria também associada ao estilo de vida de seus integrantes, seja em função dos grupos de relacionamentos sociais ou dos hábitos, comportamentos e condições de vida dos diferentes estratos da população 24 .

Inspirado na análise de Mills 32 sobre a sociedade norte-americana, Quadros 28 propõe uma estrutura sócio-ocupacional brasileira que corresponde a diferentes padrões sociais individuais captados a partir das pesquisas domiciliares do IBGE. Inicialmente, os indivíduos são classificados em classes ocupacionais segundo a ocupação do integrante familiar com o maior rendimento individual, cuja identificação é dada em termos de poder político, prestígio social e possibilidade de geração de renda da ocupação. Como descreve a Tabela 1, essa estrutura pode ser representada por 14 classes principais.
Conforme a especificidade analítica, as classes ocupacionais podem ainda ser agregadas em sete grupos ocupacionais principais: (i) empregadores [A-1 e A-2]; (ii) profissionais [C e D]; (iii) massa trabalhadora não agrícola [F, G e I]; (iv) trabalhadores não remunerados [J-1 e J-2]; (v) massa trabalhadora agrícola [H-1, H-2 e H-3]; (vi) sem ocupação sem renda [SO]; e (vii) sem ocupação com renda [SOF].

Embora as classes ocupacionais sejam um importante indicador da inserção dos indivíduos no mercado de trabalho e na sociedade, não podem ser desconsideradas significativas distinções no nível de renda que podem surgir dentro de cada um destes agrupamentos. Esta desigualdade é ainda mais acentuada pelo fato de se tratar de uma sociedade notadamente heterogênea como a brasileira, na qual, mesmo em grupos sociais com oportunidades relativamente homogêneas, é comum encontrarmos significativas distorções distributivas.

A classificação interna das classes ocupacionais segundo faixas de renda per capita cumpriria, dessa forma, um importante papel de distinção do nível econômico de famílias relativamente homogêneas segundo inserção no mercado de trabalho. Tendo como referência um valor próximo ao salário mínimo de outubro de 2007 (R\$ 380,00 ), período de referência da análise, definiram-se faixas normativas de renda familiar per capita mais ou menos condizentes com distintos padrões de vida de uma família brasileira e que, ao mesmo tempo, discriminassem parcelas diferenciadas da população, próximas aos $40 \%$ relativamente mais pobres, próximos aos $25 \%$ mais pobres, $20 \%, 10 \%$ e, finalmente, aos $5 \%$ relativamente mais ricos. Assim, a partir de valores proporcionais a $R \$ 300,00$, a população foi desagregada em cinco estratos econômicos definidos pelo rendimento per capita familiar: estrato A (acima de $\mathrm{R} \$ 1.500,00$ ), estrato $\mathrm{B}$ (entre $\mathrm{R} \$ 750,00$ e $\mathrm{R} \$$ $1.500,00$ ), estrato $\mathrm{C}$ (entre $\mathrm{R} \$ 300,00$ e $\mathrm{R} \$ 750,00$ ), estrato D (entre R\$ 150,00 e R \$ 300,00) e estrato E (abaixo de R\$ 150,00).

\section{Regressão logística}

Para avaliar de que forma as classes ocupacionais e estratos econômicos influenciam, isoladamente, o estado de saúde das pessoas, foi estabelecida uma relação logística entre a probabilidade de a pessoa declarar-se saudável e diversos fatores sócio-econômicos associados a essa condição. A relação logística, indicada para o caso de variável dependente binária (saudável e não saudável), pode ser analisada através de um modelo de regressão lógite, que se baseia na função de probabilidade logística acumulada 33 . 
Tabela 1

Tipologia de classes ocupacionais familiares segundo integrante melhor remunerado.

\begin{tabular}{|c|c|c|}
\hline Sigla & Classe ocupacional & Descrição \\
\hline A-1 & Empregadores $\geq 10$ & Famílias de empreendedores, do setor agrícola ou não agrícola, que empregam 10 ou mais ocupados \\
\hline A-2 & Empregadores $<10$ & Famílias de empreendedores, do setor agrícola ou não agrícola, que empregam menos de 10 ocupados \\
\hline C & Profissionais autônomos & $\begin{array}{l}\text { Famílias associadas às ocupações típicas de classe média, onde predominam atividades relacionadas ao } \\
\text { comércio, escritório e prestação de serviços, exercidas de forma independente pelo ocupado }\end{array}$ \\
\hline $\mathrm{D}$ & Profissionais assalariados & $\begin{array}{l}\text { Famílias associadas às profissões típicas de classe média, onde predominam atividades relacionadas ao } \\
\text { comércio, escritório e prestação de serviços, exercidas de forma assalariada pelo ocupado }\end{array}$ \\
\hline $\mathrm{F}$ & Trabalhadores autônomos & $\begin{array}{l}\text { Famílias associadas às ocupações não agrícolas de perfil operário ou assemelhado popular, que são } \\
\qquad \text { exercidas de forma independente pelo ocupado }\end{array}$ \\
\hline G & Trabalhadores assalariados & $\begin{array}{l}\text { Famílias associadas às ocupações não agrícolas de perfil operário ou assemelhado popular, que são } \\
\qquad \text { exercidas de forma assalariada pelo ocupado }\end{array}$ \\
\hline I & Trabalhadores domésticos & Famílias associadas às ocupações no serviço doméstico remunerado \\
\hline $\mathrm{J}-1$ & $\begin{array}{l}\text { Trabalhadores não remunerados } \\
\text { não agrícolas }\end{array}$ & $\begin{array}{l}\text { Famílias associadas às ocupações não remuneradas não agrícolas exercidas durante pelo menos uma hora } \\
\qquad \text { por semana em ajuda a membro do domicílio, aprendiz ou estagiário }\end{array}$ \\
\hline $\mathrm{H}-1$ & $\begin{array}{l}\text { Proprietários agrícolas } \\
\text { conta-própria }\end{array}$ & $\begin{array}{l}\text { Famílias associadas à pequena produção no ramo da agricultura ou pecuária familiar realizada sem o } \\
\qquad \text { emprego de mão-de-obra assalariada }\end{array}$ \\
\hline $\mathrm{H}-2$ & $\begin{array}{l}\text { Trabalhadores agrícolas } \\
\text { autônomos }\end{array}$ & $\begin{array}{l}\text { Famílias associadas às profissões agrícolas exercidas de forma autônoma (pescadores, caçadores, } \\
\text { extrativistas, entre outros) }\end{array}$ \\
\hline $\mathrm{H}-3$ & Assalariados agrícolas & $\begin{array}{l}\text { Famílias associadas às profissões agrícolas exercidas de forma assalariada permanente ou temporária } \\
\text { (bóia-fria) }\end{array}$ \\
\hline $\mathrm{J}-2$ & $\begin{array}{l}\text { Trabalhadores não remunerados } \\
\text { agrícolas }\end{array}$ & $\begin{array}{l}\text { Famílias associadas às ocupações não remuneradas agrícolas exercidas durante pelo menos uma hora por } \\
\text { semana, seja em atividade de autoconsumo ou em ajuda à produção familiar }\end{array}$ \\
\hline $\mathrm{SO}$ & Sem ocupação com renda & $\begin{array}{l}\text { Famílias dependentes de um membro sem ocupação na semana, mas com rendimento proveniente de } \\
\text { fonte não relacionada ao trabalho (aposentados, em sua maioria) }\end{array}$ \\
\hline SOF & Sem ocupação sem renda & Famílias sem membros ocupados e sem qualquer fonte regular de rendimento \\
\hline
\end{tabular}

Fonte: Maia 24.

Foi estimado um modelo para o ano de 1998 e outro para 2003. Para controlar a associação entre classes ocupacionais, estratos econômicos e estado de saúde dos integrantes familiares, consideraram-se as seguintes variáveis:

(a) Treze variáveis binárias para distinguir 14 classes-ocupacionais: empregadores com $10 \mathrm{em}$ pregados ou mais, empregadores com menos de 10 empregados, profissionais autônomos, profissionais assalariados, trabalhadores autônomos, trabalhadores assalariados, trabalhadores domésticos, trabalhadores agrícolas conta-própria, trabalhadores agrícolas autônomos, assalariados agrícolas, trabalhadores não remunerados não agrícolas, trabalhadores não remunerados agrícolas, sem ocupação com renda (utilizado como categoria de referência) e sem ocupação sem renda;

(b) Quatro variáveis binárias para distinguir cinco estratos econômicos: estrato A, B, C , D e E (categoria de referência);

(c) Quatro variáveis binárias para distinguir cinco regiões: Norte urbano, Sudeste, Sul, Cen-
tro-Oeste e Nordeste (categoria de referência);

(d) Uma variável binária de sexo que assume 1 para mulheres e 0 para homens;

(e) Quatro variáveis binárias para distinguir cinco categorias de cor/raça auto declarada: branca (referência), amarela, preta, parda e indígena;

(f) Décadas de idade, anos divididos por dez;

(g) Anos completos de estudo da pessoa;

(h)Uma variável binária que assume 1 quando a declaração do estado de saúde for dada pela própria pessoa e 0 quando for dada por outra pessoa.

Embora seja reconhecida a importância do efeito das interações nos diferenciais de saúde auto-avaliada, essas foram desconsideradas para simplificar a análise dos resultados e, assim, dar mais ênfase aos efeitos independentes das categorias de interesse. Por exemplo, seriam necessárias 17 variáveis adicionais apenas para considerar o efeito diferenciado do sexo sobre o estado de saúde em cada classe e estrato $\left(13^{1}+4^{1}\right)$. Analogamente, as interações entre as variáveis de controle, sexo e escolaridade, por 
exemplo, pouco modificariam os efeitos principais das classes e estratos no estado de saúde, objetivo principal deste trabalho.

Os coeficientes e variâncias dos modelos foram estimados pela rotina PROC SURVEYLOGISTIC do pacote estatístico SAS (SAS Inst., Cary, Estados Unidos). Esta rotina ajusta uma função linear para o logaritmo da razão de chances (odds ratio) pelo método de máxima verossimilhança considerando o plano amostral complexo da PNAD. As variáveis de estrutura do plano amostral dos domicílios, estratos e unidades primárias de amostragem (UPA) foram elaboradas a partir dos procedimentos sugeridos por Silva et al. 34 .

\section{Resultados}

\section{Análise descritiva}

Os resultados permitem destacar, em primeiro lugar, o baixo padrão sócio-econômico da população brasileira, refletido na expressiva participação das classes de baixo padrão social e econômico (Tabelas 2 e 3). Por exemplo, dos 176 milhões de brasileiros em 2003, 65 milhões pertenciam à massa trabalhadora não agrícola e 22 milhões à massa agrícola, além de 3,3 milhões de integrantes da classe de trabalhadores não remunerados e 4 milhões da classe dos sem ocupação sem renda. Havia, ainda, 105 milhões de pessoas com rendimento per capita inferior a $\mathrm{R} \$ 300,00$ mensais.

Os resultados também mostram uma clara hierarquia das classes ocupacionais e estratos econômicos em relação ao estado de saúde de seus integrantes (Tabela 2). Entre as classes ocupacionais, a hierarquia define-se, respectivamente, pelos empregadores, profissionais, massa não agrícola, massa agrícola e não remunerados. A prevalência de pessoas saudáveis era, por exemplo, 9 pontos percentuais superior no grupo dos empregadores em comparação à da massa agrícola em 2003 e 22 pontos superior em comparação à dos não remunerados.

A classe dos sem ocupação com renda apresentava o menor percentual de indivíduos saudáveis. A classe dos sem ocupação sem renda, composta por muitas famílias com integrantes desempregados, apresentava um percentual de pessoas saudáveis similar ao do grupo de empregadores e profissionais. Já o grupo dos trabalhadores não remunerados exibia os menores percentuais de pessoas saudáveis da estrutura social, sobretudo na classe agrícola.

Entre classes de um mesmo grupo ocupacional aparecem diferenças expressivas, como a existente entre as classes de empregadores com dez ou mais empregados em comparação àquela com menos de dez (favorável ao primeiro grupo), ou entre as classes dos trabalhadores autônomos e proprietários conta-própria agrícolas, com maior prevalência de saudáveis para os primeiros. Também chama atenção a maior prevalência de saudáveis entre os assalariados em comparação aos autônomos nos principais grupos ocupacionais. Em 2003, por exemplo, a diferença era de 4 pontos percentuais entre os profissionais e 2 pontos percentuais entre os trabalhadores não agrícolas. Entre os estratos econômicos, o percentual de indivíduos saudáveis era, por sua vez, 12 pontos percentuais superior no estrato A em comparação ao estrato E em 2003.

A dinâmica da auto-avaliação do estado de saúde entre 1998 e 2003 sugere ainda que, embora a prevalência de saudáveis permanecesse praticamente estável entre 1998 e 2003, aumentou a desigualdade entre os grupos sócio-econômicos. Reduziu o percentual de indivíduos saudáveis nos grupos mais frágeis e aumentou naqueles do topo da pirâmide social. Caiu, por exemplo, a prevalência de saudáveis na classe da massa trabalhadora agrícola, trabalhadores não remunerados, sem ocupação (com e sem renda) e nos três estratos econômicos inferiores da estrutura social. Por outro lado, aumentou na classe dos empregadores, profissionais e nos dois primeiros estratos econômicos.

A prevalência de saudáveis segundo categorias sócio-econômicas individuais (Tabela 3) permite outras importantes considerações sobre a desigualdade na auto-avaliação do estado de saúde. O percentual de pessoas saudáveis é maior para os homens, indivíduos de cor/raça branca e amarela, jovens, mais escolarizados e residentes nas regiões mais desenvolvidas do país, e reduzse substancialmente para as faixas etárias mais elevadas. Em 2003, o percentual de saudáveis entre aqueles com 9 anos de idade ou menos (91\%) era 47 pontos percentuais superior ao observado para aqueles com 60 anos de idade ou mais (44\%). As diferenças também são significativas em relação às faixas de escolaridade, chegando, em 2003, a 16 pontos percentuais entre aqueles com la a 4a a série do Ensino Fundamental (72\% de saudáveis), que apresentam o pior estado de saúde, e aqueles com superior ou mais (90\%), com o melhor estado de saúde.

O responsável pela declaração é outro importante fator a ser considerado, havendo uma expressiva superestimação do estado de saúde quando este é declarado por outra pessoa. Em 2003, por exemplo, apenas $38,2 \%$ das declarações foram dadas pela própria pessoa, sendo o percentual de saudáveis entre estes 13 pontos per- 
Distribuição da população segundo estrutura social e estado de saúde auto-avaliado. Brasil *, 1998 e 2003.

\begin{tabular}{|c|c|c|c|c|c|c|}
\hline \multirow[t]{2}{*}{ Estrutura social } & \multicolumn{3}{|c|}{1998} & \multicolumn{3}{|c|}{2003} \\
\hline & $\begin{array}{c}\text { Saudáveis } \\
(\%)\end{array}$ & $\begin{array}{c}\text { Não saudáveis } \\
\text { (\%) }\end{array}$ & $\begin{array}{c}\mathrm{n} \\
\text { (em milhares) }\end{array}$ & $\begin{array}{c}\text { Saudáveis } \\
\text { (\%) }\end{array}$ & $\begin{array}{c}\text { Não saudáveis } \\
(\%)\end{array}$ & $\begin{array}{c}\mathrm{n} \\
\text { (em milhares) }\end{array}$ \\
\hline \multicolumn{7}{|l|}{ Classes ocupacionais } \\
\hline \multicolumn{7}{|l|}{ Empregadores } \\
\hline A-1 $(>10)$ & 85,2 & 14,8 & 1.082 & 92,4 & 7,6 & 1.053 \\
\hline A-2 $(\leq 10)$ & 81,8 & 18,2 & 8.546 & 84,5 & 15,5 & 7.359 \\
\hline Total & 82,2 & 17,8 & 9.627 & 85,5 & 14,5 & 8.412 \\
\hline \multicolumn{7}{|l|}{ Profissionais } \\
\hline C (autônomos) & 80,2 & 19,8 & 8.961 & 81,9 & 18,1 & 8.306 \\
\hline D (assalariados) & 82,8 & 17,2 & 36.068 & 86,0 & 14,0 & 36.104 \\
\hline Total & 82,3 & 17,7 & 45.029 & 85,3 & 14,7 & 44.410 \\
\hline \multicolumn{7}{|l|}{ Massa trabalhadora não agrícola } \\
\hline F (autônomos) & 79,3 & 20,7 & 18.646 & 78,7 & 21,3 & 19.223 \\
\hline G (assalariados) & 80,5 & 19,5 & 34.827 & 81,1 & 18,9 & 38.618 \\
\hline I (domésticos) & 79,9 & 20,1 & 4.883 & 78,9 & 21,1 & 7.163 \\
\hline Total & 80,1 & 19,9 & 58.356 & 80,2 & 19,8 & 65.004 \\
\hline \multicolumn{7}{|l|}{ Massa trabalhadora agrícola } \\
\hline H-1 (proprietários conta-própria) & 77,3 & 22,7 & 10.377 & 74,4 & 25,6 & 10.854 \\
\hline H-2 (trabalhadores autônomos) & 81,6 & 18,4 & 1.015 & 77,8 & 22,2 & 781 \\
\hline H-3 (assalariados agrícolas) & 80,0 & 20,0 & 7.954 & 78,9 & 21,1 & 9.997 \\
\hline Total & 78,6 & 21,4 & 19.346 & 76,6 & 23,4 & 21.631 \\
\hline \multicolumn{7}{|l|}{ Trabalhadores não remunerados } \\
\hline J-1 (não agrícolas) & 75,8 & 24,2 & 473 & 74,8 & 25,2 & 369 \\
\hline J-2 (agrícolas) & 69,8 & 30,2 & 2.057 & 62,6 & 37,4 & 2.985 \\
\hline Total & 70,9 & 29,1 & 2.530 & 63,9 & 36,1 & 3.354 \\
\hline \multicolumn{7}{|l|}{ Sem ocupação } \\
\hline SO (com renda) & 67,7 & 32,3 & 19.277 & 63,5 & 36,5 & 25.319 \\
\hline SOF (sem renda) & 82,2 & 17,8 & 3.902 & 81,2 & 18,8 & 4.155 \\
\hline \multicolumn{7}{|l|}{ Estratos econômicos (Reais) ** } \\
\hline$A(>1.500)$ & 86,6 & 13,4 & 3.859 & 89,4 & 10,6 & 8.612 \\
\hline B (750-1.500) & 83,1 & 16,9 & 8.963 & 84,8 & 15,2 & 14.803 \\
\hline C (300-750) & 80,1 & 19,9 & 31.756 & 79,5 & 20,5 & 43.862 \\
\hline$D(150-300)$ & 77,0 & 23,0 & 41.384 & 74,7 & 25,3 & 44.693 \\
\hline$E(0-150)$ & 78,9 & 21,1 & 71.994 & 77,7 & 22,3 & 60.380 \\
\hline
\end{tabular}

Fonte: Pesquisa Nacional por Amostra de Domicílios (PNAD) dos anos de 1998 e 2003 (microdados); do Instituto Brasileiro de Geografia e Estatística (IBGE). Nota: porcentagens com total de $100 \%$ na linha.

* Exclusive áreas rurais dos estados de Rondônia, Acre, Amazonas, Roraima, Pará e Amapá;

** Valores de outubro de 2007 (Índice Nacional de Preços ao Consumidor corrigido para a PNAD).

centuais inferior ao das declarações dadas por outra pessoa.

Verifica-se que as desigualdades se acentuaram entre 1998 e 2003, com piora no estado de saúde, sobretudo dos menos escolarizados, mulheres e pessoas da cor/raça não branca. Na dinâmica das grandes regiões, destaca-se a redução no percentual de saudáveis nas regiões Nordeste, Sudeste e Centro-Oeste, com elevação do percentual de indivíduos saudáveis nas regiões Norte e Sul do país.

\section{$\underline{\text { Resultados da regressão logística }}$}

As razões de chances do modelo logístico permitem verificar o efeito isolado das variáveis de interesse sobre a auto-avaliação do estado de saúde das pessoas. A grande maioria das estimativas 
Tabela 3

Distribuição da população segundo características sócio-econômicas e estado de saúde auto-avaliado. Brasil *, 1998 e 2003.

\begin{tabular}{|c|c|c|c|c|c|c|}
\hline \multirow{2}{*}{$\begin{array}{l}\text { Características sócio- } \\
\text { econômicas }\end{array}$} & \multicolumn{3}{|c|}{1998} & \multicolumn{3}{|c|}{2003} \\
\hline & $\begin{array}{c}\text { Saudáveis } \\
(\%)\end{array}$ & $\begin{array}{c}\text { Não saudáveis } \\
(\%)\end{array}$ & $\begin{array}{c}\mathrm{n} \\
\text { (em milhares) }\end{array}$ & $\begin{array}{c}\text { Saudáveis } \\
\text { (\%) }\end{array}$ & $\begin{array}{c}\text { Não saudáveis } \\
(\%)\end{array}$ & $\begin{array}{c}\mathrm{n} \\
\text { (em milhares) }\end{array}$ \\
\hline \multicolumn{7}{|l|}{ Declaração } \\
\hline Própria pessoa & 70,3 & 29,7 & 58.197 & 70,3 & 29,7 & 67.209 \\
\hline Outra pessoa & 84,2 & 15,8 & 99.949 & 83,7 & 16,3 & 108.717 \\
\hline \multicolumn{7}{|l|}{ Sexo } \\
\hline Masculino & 81,8 & 18,2 & 77.353 & 81,0 & 19,0 & 85.822 \\
\hline Feminino & 76,4 & 23,6 & 80.793 & 76,3 & 23,7 & 90.104 \\
\hline \multicolumn{7}{|l|}{ Cor/raça } \\
\hline Branca & 80,5 & 19,5 & 84.048 & 80,6 & 19,4 & 91.354 \\
\hline Preta & 76,8 & 23,2 & 8.998 & 75,4 & 24,6 & 10.416 \\
\hline Amarela & 83,2 & 16,8 & 821 & 80,1 & 19,9 & 766 \\
\hline Parda & 77,4 & 22,6 & 63.898 & 76,5 & 23,5 & 73.050 \\
\hline Indígena & 80,8 & 19,2 & 365 & 72,9 & 27,1 & 332 \\
\hline \multicolumn{7}{|l|}{ Faixa etária (anos) } \\
\hline $0-9$ & 91,7 & 8,3 & 30.492 & 90,8 & 9,2 & 31.358 \\
\hline $10-19$ & 91,8 & 8,2 & 33.948 & 91,1 & 8,9 & 33.941 \\
\hline $20-29$ & 86,3 & 13,7 & 26.101 & 86,3 & 13,7 & 31.124 \\
\hline $30-39$ & 79,9 & 20,1 & 23.511 & 79,8 & 20,2 & 26.022 \\
\hline $40-49$ & 68,9 & 31,1 & 18.345 & 70,2 & 29,8 & 21.794 \\
\hline $50-59$ & 54,7 & 45,3 & 11.908 & 58,0 & 42,0 & 14.754 \\
\hline 60 ou mais & 39,3 & 60,7 & 13.828 & 43,5 & 56,5 & 16.903 \\
\hline \multicolumn{7}{|l|}{ Escolaridade } \\
\hline Sem escolaridade & 75,1 & 24,9 & 43.445 & 74,0 & 26,0 & 42.003 \\
\hline 1a a $4 \underline{\mathrm{a}}$ série & 74,7 & 25,3 & 47.915 & 72,1 & 27,9 & 46.799 \\
\hline $5 \underline{a}$ a $8 \underline{a}$ série & 82,5 & 17,5 & 35.569 & 81,2 & 18,8 & 40.594 \\
\hline Ensino Médio & 86,4 & 13,6 & 21.515 & 86,0 & 14,0 & 33.015 \\
\hline Ensino Superior & 89,8 & 10,2 & 9.165 & 89,9 & 10,1 & 12.712 \\
\hline \multicolumn{7}{|l|}{ Grande região } \\
\hline Norte & 73,9 & 26,1 & 9.008 & 76,3 & 23,7 & 10.561 \\
\hline Nordeste & 76,8 & 23,2 & 47.272 & 74,6 & 25,4 & 50.468 \\
\hline Sudeste & 81,6 & 18,4 & 65.973 & 81,4 & 18,6 & 75.979 \\
\hline Sul & 79,1 & 20,9 & 23.936 & 79,4 & 20,6 & 26.293 \\
\hline Centro-Oeste & 78,1 & 21,9 & 11.957 & 77,5 & 22,5 & 12.626 \\
\hline
\end{tabular}

Fonte: Pesquisa Nacional por Amostra de Domicílios (PNAD) dos anos de 1998 e 2003 (microdados); do Instituto Brasileiro de Geografia e Estatística (IBGE). Nota: porcentagens com total de $100 \%$ na linha.

* Exclusive áreas rurais dos estados de Rondônia, Acre, Amazonas, Roraima, Pará e Amapá.

obtidas pelo método de máxima verossimilhança para os anos de 1998 e 2003 mostrou-se significativa supondo um intervalo de $95 \%$ de confiança (Tabela 4). O dois períodos apresentaram ainda satisfatórias estatísticas de qualidade do ajuste, com percentuais de concordância equivalentes a $77 \%$ e coeficientes de determinação ajustados equivalentes a $22 \%$ e $21 \%$, respectivamente, para 1998 e 2003.
Independente das variáveis de controle, as classes ocupacionais e os estratos econômicos cumprem um importante papel na definição do estado de saúde das pessoas. Um resultado que chama atenção é o fato da probabilidade de declarar-se saudável ser maior nas classes agrícolas em comparação às não agrícolas. Em 2003, as razões de chances de ser saudável entre os trabalhadores agrícolas eram iguais ou superiores a 
Estimativas de máxima verossimilhança para razão de chances (RC) de ser saudável e intervalo de 95\% de confiança (IC95\%). Brasil * 1998 e 2003.

\begin{tabular}{|c|c|c|c|c|}
\hline \multirow[t]{2}{*}{ Variável } & \multicolumn{2}{|c|}{1998} & \multicolumn{2}{|c|}{2003} \\
\hline & $\mathrm{RC}$ & IC95\% & RC & IC95\% \\
\hline \multicolumn{5}{|l|}{ Classe Ocupacional } \\
\hline A-1 (Empregadores > 10) & 1,167 & $0,992-1,372$ & 1,740 & $1,370-2,211$ \\
\hline A-2 (Empregadores $\geq 10$ ) & 1,153 & $1,075-1,236$ & 1,220 & $1,132-1,316$ \\
\hline C (Profissionais autônomos) & 1,149 & $1,080-1,223$ & 1,202 & $1,126-1,283$ \\
\hline D (Profissionais assalariados) & 1,195 & $1,142-1,250$ & 1,341 & $1,284-1,402$ \\
\hline F (Trabalhadores não agrícolas autônomos) & 1,126 & $1,071-1,184$ & 1,149 & $1,097-1,203$ \\
\hline G (Trabalhadores não agrícolas assalariados) & 1,136 & $1,085-1,190$ & 1,190 & $1,141-1,240$ \\
\hline I (Trabalhadores domésticos) & 1,171 & $1,081-1,268$ & 1,166 & $1,090-1,246$ \\
\hline H-1 (Proprietários agrícolas conta-própria) & 1,327 & $1,224-1,438$ & 1,357 & $1,219-1,510$ \\
\hline H-2 (Trabalhadores agrícolas autônomos) & 1,518 & $1,260-1,830$ & 1,370 & $1,046-1,795$ \\
\hline H-3 (Trabalhadores agrícolas assalariados) & 1,285 & $1,189-1,390$ & 1,331 & $1,237-1,433$ \\
\hline J-1 (Trabalhadores não remunerados não agrícolas) & 0,967 & $0,763-1,225$ & 1,128 & $0,920-1,384$ \\
\hline J-2 (Trabalhadores não remunerados agrícolas) & 1,114 & $0,999-1,242$ & 1,032 & $0,935-1,139$ \\
\hline SOF (Sem ocupação sem renda) & 1,068 & $0,980-1,163$ & 1,135 & $1,038-1,241$ \\
\hline \multicolumn{5}{|l|}{ Estrato econômico (Reais) } \\
\hline$A(>1.500)$ & 1,891 & $1,717-2,083$ & 3,238 & $2,987-3,512$ \\
\hline$B(750-1.500)$ & 1,465 & $1,372-1,564$ & 2,143 & $2,016-2,279$ \\
\hline$C(300-750)$ & 1,231 & $1,179-1,286$ & 1,537 & $1,478-1,600$ \\
\hline$D(150-300)$ & 1,086 & $1,049-1,126$ & 1,158 & $1,117-1,201$ \\
\hline \multicolumn{5}{|l|}{ Grande região } \\
\hline Norte & 0,677 & $0,625-0,735$ & 0,856 & $0,777-0,944$ \\
\hline Sudeste & 1,277 & $1,206-1,352$ & 1,350 & $1,267-1,440$ \\
\hline Sul & 1,041 & $0,972-1,115$ & 1,122 & $1,034-1,218$ \\
\hline Centro-Oeste & 0,912 & $0,853-0,974$ & 0,924 & $0,858-0,995$ \\
\hline Feminino & 0,738 & $0,723-0,753$ & 0,797 & $0,782-0,811$ \\
\hline \multicolumn{5}{|l|}{ Cor/raça } \\
\hline Amarela & 1,417 & $1,138-1,765$ & 1,058 & $0,831-1,348$ \\
\hline Preta & 0,975 & 0,918-1,035 & 0,949 & $0,902-0,998$ \\
\hline Parda & 0,864 & $0,835-0,894$ & 0,898 & $0,869-0,928$ \\
\hline Indígena & 0,974 & 0,730-1,301 & 0,837 & $0,620-1,130$ \\
\hline Idade (décadas) & 0,606 & $0,602-0,611$ & 0,624 & $0,620-0,629$ \\
\hline Anos de estudo & 1,093 & $1,090-1,097$ & 1,066 & $1,063-1,069$ \\
\hline Próprio declarante & 0,866 & $0,846-0,887$ & 0,899 & $0,878-0,921$ \\
\hline
\end{tabular}

Fonte: Pesquisa Nacional por Amostra de Domicílios (PNAD) dos anos de 1998 e 2003 (microdados); do Instituto Brasileiro de Geografia e Estatística (IBGE).

* Exclusive áreas rurais dos estados de Rondônia, Acre, Amazonas, Roraima, Pará e Amapá.

$33 \%$ em relação àquela observada na classe dos indivíduos sem ocupação com renda (categoria de referência). Nas classes tipicamente não agrícolas, apenas profissionais assalariados e empregadores com 10 ou mais empregados apresentavam razões iguais ou superiores a estas.

Também se confirma, entre integrantes de um mesmo grupo ocupacional, o melhor estado de saúde nas classes assalariadas. Por exemplo, a razão de chances de ser saudável era, em 2003, 14 pontos percentuais superior entre os profissionais assalariados quando comparada à dos profissionais autônomos. Entre os trabalhadores não agrícolas, a mesma relação era 4 pontos percentuais superior para os assalariados.

Os coeficientes associados aos estratos econômicos mostram que, mesmo isolando o efeito das variáveis de controle, mantém-se uma clara 
hierarquia em relação ao estado de saúde. Em 2003, a propensão a declarar-se saudável entre os integrantes do estrato A era mais que três vezes superior à daqueles da classe E (224\%). Embora se reduza mais da metade, a diferença das propensões permanece expressiva entre integrantes dos estratos B e E (102\%) e é significativa mesmo entre integrantes dos estratos $\mathrm{D}$ e E (16\%).

Em relação às características sócio-econômicas individuais, a probabilidade de declarar-se saudável é maior entre moradores das regiões Sudeste e Sul, respectivamente, independente das demais características consideradas. O caso extremo inferior passa a ocorrer na Região Norte, onde a razão de chances dos moradores se declararem com bom estado de saúde era, em 2003, 14\% inferior à dos moradores da Região Nordeste.

Homens declaram melhor estado de saúde, com uma razão de chances $20 \%$ superior às mulheres. Por outro lado, as diferenças entre cor/ raça deixam de ser expressivas quando considerada a posição social das pessoas. Em 2003, a diferença para a razão de chances daqueles de cor branca mostrou-se significativa apenas em comparação àqueles da cor preta e parda, com razões de chances, respectivamente, $5 \%$ e $10 \%$ inferiores à dos brancos.

A probabilidade de declarar-se saudável está, ainda, negativamente associada à idade da pessoa (redução de $38 \%$ na razão de chances, em 2003, para cada década adicional de idade) e positivamente associada à escolaridade (crescimento de $7 \%$ na razão de chances para cada ano adicional de escolaridade). A avaliação do estado de saúde também depende do responsável pela declaração, com uma razão de chances $10 \%$ inferior de apresentar boa saúde quando o informante da resposta é o próprio entrevistado.

Comparando os coeficientes estimados para os anos de 1998 e 2003 é possível compreender a dinâmica da desigualdade entre as diferentes categorias de análise. Por exemplo, aumentaram as diferenças das declarações de boa saúde entre os estratos econômicos, aquelas da classe sem ocupação com renda e praticamente todas as demais classes ocupacionais.

A redução mais acentuada na probabilidade de declarar-se saudável na Região Nordeste seria uma das principais responsáveis pelo crescimento da diferenciação desta região em relação às mais desenvolvidas, Sudeste e Sul. Outro fato relevante é a redução do fator idade e escolaridade na probabilidade de declarar-se saudável, com melhor estado de saúde para os mais idosos e menos escolarizados.

\section{Discussão}

Este trabalho verificou de que maneira a posição social influencia o estado de saúde dos brasileiros a partir de medidas de saúde auto-reportadas do suplemento de saúde da PNAD de 1998 e 2003. A análise baseou-se em um método de estratificação das famílias que considera a posição dos integrantes familiares no mercado de trabalho (classes ocupacionais) e os estratos econômicos definidos pelo rendimento familiar per capita.

A hipótese é que a posição das famílias na estrutura de uma sociedade funcionaria como uma proxy para os diferentes estilos de vida da população, com impactos sobre os hábitos, condições de vida, comportamentos para uma vida mais saudável e, conseqüentemente, sobre a saúde dos integrantes familiares. A posição social refletiria, assim, desde as características do ambiente psicossocial do trabalho 21 , disponibilidade de investimento em insumos de saúde, como cuidados médicos ou alimentação, até comportamentos que melhoram a qualidade de vida.

Entre algumas das propostas de estratificação da sociedade brasileira $27,28,35$, a estrutura social adotada neste trabalho, definida pela conjugação entre as classes ocupacionais e os estratos econômicos, cumpriria um importante papel na definição dos grupos sócio-econômicos razoavelmente consistentes com os complexos padrões sociais brasileiros 24,36 . Seria, assim, um importante preditor das condições de saúde da população brasileira.

O estado de saúde foi mensurado a partir da variável de saúde auto-reportada. Esta medida tem sido uma das mais utilizadas na literatura empírica para comparação da saúde entre distintos grupos populacionais, por ser uma medida do estado de saúde que não se limita a nenhuma doença ou estado funcional específico ${ }^{37}$. Por este motivo, consegue captar, de forma ampla, o estado de saúde individual, que depende tanto do conhecimento acerca dos fatores de risco e diagnósticos de saúde quanto do grau de satisfação do indivíduo com o ambiente social em que está inserido. Diversos estudos têm mostrado que essa variável é útil para complementar diagnósticos médicos e mostrar a aderência a certos tratamentos de saúde, uma vez que determinadas sensações do paciente não são captadas em exames usuais 38,39 . Outros mostram que ela é melhor para prever a mortalidade do que testes clínicos, controlando por doenças crônicas e limitações funcionais 40 .

No entanto, esta medida não está isenta de limitações, que devem ser consideradas na interpretação dos resultados. Uma delas refere-se ao desconhecimento dos grupos de referência 
que os indivíduos usam para comparações 41 . Neste caso, as diferenças de saúde podem não ter respaldo em indicadores objetivos do estado de saúde, mas representar apenas expectativas e visões distintas do mesmo problema entre os grupos. Como não é possível superar essa lacuna com os dados disponíveis, selecionamos variáveis demográficas e sócio-econômicas usualmente encontradas na literatura para comparação social 41 .

Outro problema da análise refere-se ao responsável pelas declarações. Como não é possível garantir a presença de todos os moradores do domicílio no momento da entrevista, a avaliação daqueles ausentes acaba sendo subjetivamente feita por parte de outra pessoa. Nessas situações, constatou-se que há uma superestimação do estado de saúde e, dada a expressiva participação daqueles que não declaram o próprio estado de saúde na PNAD, há uma conseqüente superestimação das condições de saúde da população brasileira.

Os resultados corroboram a literatura sobre o tema ao apontar uma relação positiva do estado de saúde com indicadores sócio-econômicos como renda e escolaridade e negativa com a idade da pessoa 7,19,20. Além disso, também comprova trabalhos que mostram que a saúde é pior para as minorias raciais e mulheres 42 , embora as diferenças de cor/raça se reduzam sensivelmente quando consideradas as posições sociais dos indivíduos. Essas diferenças no estado de saúde entre grupos de cor/raça podem associar-se a características sócio-econômicas, acesso diferenciado de bens e serviços de saúde na sociedade 43 , à discriminação racial e diferenças genéticas na predisposição a doenças para indivíduos de diferentes grupos raciais/étnicos 44 . O fato de as mulheres declararem pior estado de saúde pode expressar distintas características hormonais e biológicas e a percepções mais apuradas do estado de saúde, além de mais facilidade para relatar problemas do que homens 45,46 .

Entre indivíduos de um mesmo grupo ocupacional, os assalariados possuem melhor estado de saúde em relação aos autônomos. O assalariamento aumentaria o poder e a independência econômica, além de conferir recompensas não econômicas como suporte social e reconhecimento de outras pessoas 47 . Por outro lado, a instabilidade social provocada por fatores associados a muitas posições autônomas poderia ter efeitos deletérios sobre a saúde. Estudos mostram que a insegurança financeira está associada à pior auto-avaliação do estado de saúde, maior incidência de depressão e aumento da pressão sanguínea 48 .
Contrário a outros trabalhos 49 , os resultados mostram ainda que, embora o percentual de saudáveis seja maior nas classes não agrícolas, a probabilidade de uma pessoa declarar-se saudável é maior para as classes agrícolas quando isolados os efeitos das variáveis de controle. Segundo Corin 50, a vida no campo pode ser tanto protetora quanto desgastante, resultando em efeitos contraditórios do trabalho agrícola e da vida rural sobre a saúde. Os efeitos do comportamento individual sobre a saúde dependem, em grande medida, da forma como as relações sociais são estabelecidas dentro dessa coletividade. A maior probabilidade de declarar-se saudável para trabalhadores agrícolas pode refletir falta de informação e baixas expectativas em relação à saúde dessas pessoas 7, ou ainda menor acesso aos serviços de saúde, quando comparados a residentes de áreas urbanas, o que faz com que eles desconheçam um possível diagnóstico desfavorável à saúde 51 .

Entretanto, estudos apontam que as grandes diferenças observadas na saúde da população em geral, e de trabalhadores, em particular, não encontram muita explicação na disponibilidade de serviços de saúde 52 . As razões pelas quais a posição na hierarquia social afeta a saúde ocorrem principalmente por mecanismos psiconeuro-endócrinos. Situações de ansiedade no trabalho, por exemplo, fazem com que o cérebro concentre os esforços na superação do estresse, deixando de lado funções necessárias ao crescimento e digestão 53. Embora não seja possível elucidar os motivos das desigualdades em saúde na hierarquia social com os dados deste trabalho, é importante analisar a magnitude das diferenças entre grupos.

\section{Considerações finais}

De maneira geral, os resultados corroboram a literatura ao apontar que, independente de características sócio-demográficas individuais, a posição social cumpre um importante papel na distinção da percepção de saúde da população brasileira, não importa qual o indicador utilizado para distinguir os grupos sociais 54 .

A complexidade do tema acaba impondo sérias limitações à análise. O indicador de posição social construído representa uma das inúmeras tipologias de estratificação social que podem ser analisadas. Qualquer tentativa de reproduzir a complexa estrutura de classes de uma sociedade em algumas categorias analíticas provocará uma indubitável perda de informação. Algumas classes mereceriam um estudo mais apurado sobre o estado de saúde de seus integrantes, que consi- 
derasse a heterogeneidade de suas composições. Além disso, não se sabe se as evidências encontradas permaneceriam caso outras medidas de saúde fossem consideradas.

Apesar das limitações, esse estudo vai além dos trabalhos usualmente encontrados sobre a relação entre características sócio-econômicas e saúde, ao incorporar aspectos relacionados a padrões e estilos de vida de diferentes grupos ocupacionais. Análises mais detalhadas seriam necessárias para ajudar a elucidar os motivos das diferenças na percepção de saúde entre os grupos, o que poderia servir de subsídio para intervenções focadas na saúde do trabalhador.

\section{Resumo}

O objetivo deste trabalho é analisar de que forma a posição social das famílias está associada ao estado de saúde individual auto-avaliado. As informações foram extraídas dos suplementos de saúde da Pesquisa Nacional por Amostra de Domicílios (PNAD/IBGE) de 1998 e 2003. A análise baseou-se em estatísticas descritivas e regressão logística para captar a relação condicional entre estado de saúde, posição social e demais variáveis de controle, como idade, sexo, raça/cor, renda, escolaridade e região de residência. Os resultados mostram que a hierarquia de classes ocupacionais reproduz-se na auto-avaliação do estado de saúde. Há um aumento das desigualdades entre 1998 e 2003, com redução na prevalência de saudáveis nos grupos de base e aumento no topo da pirâmide social. Embora a prevalência de saudáveis seja maior nas classes não agrícolas, a probabilidade de declarar-se saudável é maior para os agrícolas, depois de adicionadas as demais variáveis de controle. Os resultados reforçam a necessidade de se analisar as desigualdades em saúde sob a perspectiva da estratificação social.

Classe Social; Auto-Avaliação da Saúde; Desigualdades em Saúde

\section{Colaboradores}

Ambos os autores participaram de todas as etapas deste trabalho. 


\section{Referências}

1. Strauss J, Thomas D. Health, nutrition, and economic development. J Econ Lit 1998; 36:766-817.

2. Grossman M. The human capital model. In: Culyer AJ, Newhouse JP, editors. Handbook of health economics. Amsterdam: Elsevier; 2000. p. 347-408.

3. Brito JC. Enfoque de gênero e relação saúde/trabalho no contexto de reestruturação produtiva e precarização do trabalho. Cad Saúde Pública 2000; 16:195-204.

4. Salim CA. Doenças do trabalho: exclusão, segregação e relações de gênero. São Paulo Perspect 2003; 17:11-24.

5. Pol LG, Thomas RK. The demography of health and health care. 2nd Ed. New York: Plenum Press; 2001.

6. Ludermir AB, Melo Filho DA. Condições de vida e estrutura ocupacional associadas a transtornos mentais comuns. Rev Saúde Pública 2002; 36: 213-21.

7. Comissão Nacional sobre Determinantes Sociais da Saúde. As causas das iniqüidades em saúde no Brasil. Rio de Janeiro: Comissão Nacional sobre Determinantes Sociais da Saúde; 2008.

8. Wilkinson R, Marmot M. Social determinants of health: the solid facts. http://www.euro.who. int/DOCUMENT/E81384.pdf (acessado em 04/ Jan/2010).

9. Buss PM, Pellegrini Filho A. A saúde e seus determinantes sociais. Physis (Rio J.) 2007; 17:77-93.

10. Barata RB, Almeida MF, Monteiro CV, Silva ZP. Health inequalities based on ethnicity in individuals aged 15 to 64, Brazil, 1998. Cad Saúde Pública 2007; 23:305-13.

11. Dachs JNW. Determinantes das desigualdades na auto-avaliação do estado de saúde no Brasil: análise dos dados da PNAD/1998. Ciênc Saúde Coletiva 2002; 7:641-57.

12. Neri M, Soares WL, Soares C. Condições de saúde no setor de transporte rodoviário de cargas e de passageiros: um estudo baseado na Pesquisa $\mathrm{Na}$ cional por Amostra de Domicílios. Cad Saúde Pública 2005; 21:1107-23.

13. Stansfeld SA, North FM, White I, Marmot MG. Work characteristics and psychiatric disorder in civil servants in London. J Epidemiol Community Health 1995; 49:48-53.

14. Wilkinson RG. The epidemiological transition. In: Kawachi I, Kennedy BP, Wilkinson RG, editors. The society and population health reader. v. 1: income inequality and health. New York: New Press; 1999. p. 36-46.

15. Lynch JW, Kaplan GA, Pamuk ER, Cohen RD, Heck $\mathrm{KE}$, Balfour JL, et al. Income inequality and mortality in metropolitan areas of the United States. Am J Public Health 1998; 88:1074-80.

16. Kawachi I, Kennedy BP. Health and social cohesion: why care about income inequality? In: Kawachi I, Kennedy BP, Wilkinson RG, editors. The society and population health reader. v. 1: income inequality and health. New York: New Press; 1999. p. 195-201.
17. Wilson M, Daly N. Life expectancy, economic inequality, homicide, and reproductive timing in Chicago neighborhoods. In: Kawachi I, Kennedy BP, Wilkinson RG, editors. The society and population health reader. v. 1: income inequality and health. New York: New Press; 1999. p. 297-304.

18. Deaton AS, Paxson $\mathrm{CH}$. Aging and inequality in income and health. Am Econ Rev 1998; 88:248-53.

19. Kassouf AL. Rendimentos perdidos por trabalhadores em condições inadequadas de saúde. Economia Aplicada 1999; 3:239-62.

20. Alves LF. Impactos do estado de saúde sobre os rendimentos individuais no Brasil [Dissertação de Mestrado]. Belo Horizonte: Centro de Desenvolvimento e Planejamento Regional, Universidade Federal de Minas Gerais; 2002.

21. Mustard CA, Vermeulen M, Lavis JN. Is the position in the occupational hierarchy a determinant of decline in perceived health status? Soc Sci Med 2003; 57:2291-303.

22. Volkers AC, Westert GP, Schellevis FG. Health disparities by occupation, modified by education: a cross-sectional population study. BMC Public Health 2007; 7:1-10.

23. Collins AM, Goldman N. Perceived social position and health in older adults. Princeton: University of Princeton; 2006. (Working Paper, 2006-08).

24. Maia AG. Estrutura de classes e desigualdades no Brasil. São Paulo: LTr; 2009.

25. Rose D, Pevalin DJ. The national statistics socioeconomic classification: unifying official and sociological approaches to the conceptualization and measurement of social class. Colchester: University of Essex; 2001. (ISER Working Papers, 4).

26. Wright EO. Classes. New York: Editora Verso; 1985.

27. Valle-Silva N. Atualização da escala sócio-econômica de ocupações para 1980. Rio de Janeiro: Laboratório Nacional de Computação Científica; 1985.

28. Quadros WJ. A evolução da estrutura social brasileira: notas metodológicas. Campinas: Instituto de Economia, Universidade Estadual de Campinas; 2008. (Texto para Discussão, 147).

29. Williams DR, Collins C. US socioeconomic and racial differences in health: patterns and explanations. Annu Rev Sociol 1995; 21:349-86.

30. Noronha KVMS, Andrade MV. O efeito da distribuição de renda sobre o estado de saúde individual no Brasil. Pesquisa e Planejamento Econômico 2007; 37:521-56.

31. Andrade MV. A saúde na PNAD. Belo Horizonte: Centro de Desenvolvimento e Planejamento Regional, Universidade Federal de Minas Gerais; 2002. (Texto para Discussão, 170).

32. Mills W. A nova classe média. 3a Ed. Rio de Janeiro: Zahar Editores; 1979.

33. Pindyck RS, Rubinfeld DL. Econometria: modelos e previsões. 4a Ed. Rio de Janeiro: Elsevier; 2004.

34. Silva PLN, Pessoa DGC, Lila MF. Análise estatística de dados da PNAD: incorporando a estrutura do plano amostral. Ciênc Saúde Coletiva 2002; 7: 659-70. 
35. Figueiredo-Santos JAF. Estrutura de posições de classe no Brasil: mapeamento, mudanças e efeitos na renda. Belo Horizonte: Editora UFMG; 2002.

36. Maia AG, Quadros WJ. Tipologia municipal de classes sociocupacionais: uma nova dimensão para análise das desigualdades territoriais no Brasil. Revista de Economia e Sociologia Rural 2009; 47:389-418.

37. Jylha M. What is self-rated health and why does it predict mortality? Towards a unified conceptual model. Soc Sci Med 2009; 69:307-16.

38. Wang C, Satariano WA. Self-rated current and future health independently predict subsequent mortality in an aging population. J Gerontol A Biol Sci Med Sci 2007; 62:1428-34.

39. Pocock SJ, Henderson RA, Clayton T, Lyman GH, Chamberlain DA. Quality of life after coronary angioplasty or continued medical treatment for angina: three-year follow-up in the RITA-2 trial. J Am Coll Cardiol 2000; 35:907-14.

40. Shadbolt B, Barresi J, Craft P. Self-rated health as a predictor of survival among patients with advanced cancer. J Clin Oncol 2002; 20:2514-9.

41. Subramanyam M, Kawachi I, Berkman L, Subramanian SV. Relative deprivation in income and self-rated health in the United States. Soc Sci Med 2009; 69:327-34.

42. Kahn JR, Fazio EM. Economic status over the life course and racial disparities in health. J Gerontol 2005; 60B:76-84.

43. Bastos JF, Peres MA, Peres KG, Dumith SC, Gigante DP. Diferenças socioeconômicas entre autoclassificação e heteroclassificação de cor/raça. Rev Saúde Pública 2008; 42:324-34.

44. Collins FS. What we do and don't know about 'race', 'ethnicity', genetics and health at the dawn of the genome era. Nat Genet 2004; 36(11 Suppl):S13-5.

45. Macintyre S, Hunt K, Sweeting H. Gender differences in health: are things really as simple as they seem? Soc Sci Med 1996; 42:617-24.
46. Mendoza-Sassi RA, Béria JU. Gender differences in self-reported morbidity: evidence from a population-based study in southern Brazil. Cad Saúde Pública 2007; 23:341-6.

47. Ross C, Mirowsky J. Does employment affect health? J Health Soc Behav 1995; 36:230-40.

48. Ferrie JE, Shipley MJ, Stansfeld SA, Smith GD, Marmot M. Future uncertainty and socioeconomic inequalities in health: the Whitehall II study. Soc Sci Med 2003; 57:637-46.

49. Gueorguieva R, Sindelar JL, Falba TA, Fletcher JM, Keenan P, Wu R, et al. The impact of occupation on self-rated health: cross-sectional and longitudinal evidence from the health and retirement survey. J Gerontol B Psychol Sci Soc Sci 2009; 64:118-24.

50. Corin E. The social and cultural matrix of health and disease. In: Evans RG, Barer ML, Marmor TR, editors. Why are some people healthy and others not? New York: Aldine de Gruyter; 1994. p. 93-132.

51. Zhang P, Tao G, Anderson LA. Differences in access to health care services among adults in rural America by rural classification categories and age. Aust J Rural Health 2008; 11:64-72.

52. Evans RG. Introduction. In: Evans RG, Barer ML, Marmor TR, editors. Why are some people healthy and others not? New York: Aldine de Gruyter; 1994. p. 3-26.

53. Kivimäki M, Vahtera J, Elovainio M, KeltikangasJärvinen L, Virtanen M, Hintsanen M, et al. What are the next steps for research on work stress and coronary heart disease? 2008; SJWEH Supplements 2008; (6):33-40.

54. Hertzman C, Frank J, Evans RG. Heterogeneities in health status and the determinants of population health. In: Evans RG, Barer ML, Marmor TR, editors. Why are some people healthy and others not? New York: Aldine de Gruyter; 1994. p. 67-92.

Recebido em 05/Ago/2009

Versão final reapresentada em 21/Jan/2010 Aprovado em 22/Fev/2010 